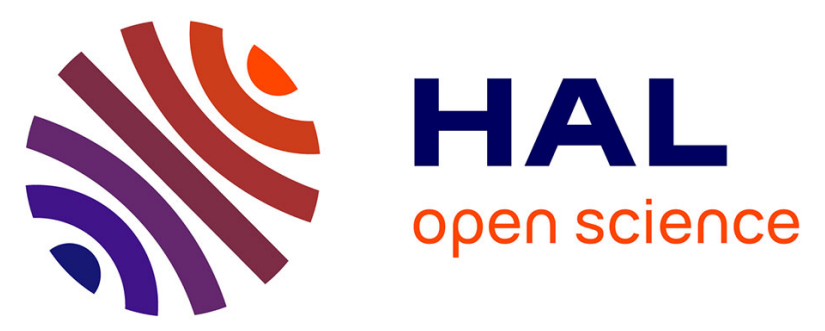

\title{
Using Density Functional Theory Based Methods to Investigate the Photophysics of Polycyclic Aromatic Hydrocarbon Radical Cations: A Benchmark Study on Naphthalene, Pyrene and Perylene Cations
}

\author{
Martial Boggio-Pasqua, Michael J. Bearpark
}

\section{To cite this version:}

Martial Boggio-Pasqua, Michael J. Bearpark. Using Density Functional Theory Based Methods to Investigate the Photophysics of Polycyclic Aromatic Hydrocarbon Radical Cations: A Benchmark Study on Naphthalene, Pyrene and Perylene Cations. ChemPhotoChem, 2019, 3 (9), pp.763-769. 10.1002/cptc.201900130 . hal-02310866

\section{HAL Id: hal-02310866 \\ https://hal.science/hal-02310866}

Submitted on 15 Jun 2020

HAL is a multi-disciplinary open access archive for the deposit and dissemination of scientific research documents, whether they are published or not. The documents may come from teaching and research institutions in France or abroad, or from public or private research centers.
L'archive ouverte pluridisciplinaire HAL, est destinée au dépôt et à la diffusion de documents scientifiques de niveau recherche, publiés ou non, émanant des établissements d'enseignement et de recherche français ou étrangers, des laboratoires publics ou privés. 


\title{
On the Use of Density Functional Theory-Based Methods to
} Investigate the Photophysics of Polycyclic Aromatic Hydrocarbon Radical Cations: A Benchmark Study on Naphthalene, Pyrene and Perylene Cations.

\author{
Martial Boggio-Pasqua ${ }^{*[a]}$, Michael J. Bearpark ${ }^{[\mathrm{b}]}$
}

\begin{abstract}
Unrestricted DFT (UDFT), time-dependent DFT (TDDFT) and spin-flip TDDFT (SF-TDDFT) were used to investigate the potential energy surfaces of the ground and first two electronic excited states of the naphthalene, pyrene and perylene radical cations. In particular, conical intersections - which play a central role in the photophysics of these cations - were located with these DFTbased methods. The results are consistent with accurate multiconfigurational wavefunction-based ab initio methods. These show that naphthalene and pyrene cations can quickly relax nonradiatively from their excited states back down to the original ground state species through easily accessible conical intersections, but the perylene cation cannot do so, due to the absence of any accessible funnels between the lowest excited state and the ground state, leaving radiative decay as the most probable photophysical pathway. This study paves the way for using computationally efficient density functional theory (DFT)-based methods in future investigations of the photophysics of much larger polycyclic aromatic hydrocarbons, for which multiconfigurational wavefunction-based methods become prohibitively expensive.
\end{abstract}

\section{Introduction}

Monocations of polycyclic aromatic hydrocarbons (PAHs) have generated considerable interest in the astrophysics community, due to their relevance in models accounting for observed spectral absorption and emission features of the interstellar medium (ISM). ${ }^{[1]}$ In particular, small $\mathrm{PAH}$ cations such as naphthalene $\left(\mathrm{Nap}^{\circ+}\right)$ and pyrene $\left(\mathrm{Pyr}^{*+}\right)$ have often been proposed as candidates for carriers of diffuse interstellar absorption bands (DIBs). ${ }^{[2,3]}$ These two cations have very short excited-state lifetimes associated with subpicosecond groundstate recovery, ${ }^{[4,5]}$ while perylene $\left(\mathrm{Per}^{{ }^{+}}\right)$is considered to be the first fluorescent PAH cation, ${ }^{[6]}$ for which a much longer excitedstate lifetime has been observed. ${ }^{[4]}$ Thus, these PAH cations present very distinct photophysical behaviors, which have first

[a] Dr, M., Boggio-Pasqua

Laboratoire de Chimie et Physique Quantiques

CNRS et Université Toulouse III - Paul Sabatier

118 route de Narbonne, 31062 Toulouse, France

E-mail: martial.boggio@irsamc.ups-tlse.fr

[b] Pr, M., J., Bearpark

Department of Chemistry, Molecular Sciences Research Hub Imperial College London, White City Campus

80 Wood Lane, London W12 0BZ, UK

E-mail: m.bearpark@imperial.ac.uk been rationalized based on investigation of the static potential energy surfaces (PESs) involved.

The ultrafast ground-state recovery of $\mathrm{Nap}^{\circ+}$ and $\mathrm{Pyr}^{\circ+}$ was explained by easily accessible sloped conical intersections providing very efficient nonradiative decay paths from the initially excited $D_{2}$ state down to $D_{0} .^{[7,8]}$ On the other hand, only inaccessible high-energy conical intersections could be located between the ground and first excited states in Per ${ }^{\circ+}$, providing a simple explanation for its longer excited-state lifetime and fluorescent behavior. ${ }^{[9]}$ These findings were based on accurate but computationally highly demanding wavefunction-based approaches such as the complete active space self-consistent field (CASSCF) method together with complete active space second-order perturbation theory (CASPT2). These methods, while applicable for $\mathrm{Nap}^{\circ+}$ and $\mathrm{Pyr}^{\circ+}$, become prohibitively expensive for Per*t for which further approximations had to be introduced by using a restricted active space (RASSCF) wavefunction. ${ }^{[10]}$

To investigate excited electronic states of the many larger PAH cations that are potentially relevant for molecular astrophysics such as coronene or corannulene ${ }^{[11]}$ - more computationally affordable quantum chemical methods are desirable, provided that they can describe the relevant potential energy surfaces and critical conical intersections between them realistically. In this article, we therefore revisit the photophysical pathways of $\mathrm{Nap}^{*+}$, $\mathrm{Pyr}^{\cdot+}$ and $\mathrm{Per}^{*+}$ using density functional theory (DFT)based approaches: unrestricted DFT (UDFT), time-dependent DFT (TDDFT) and spin-flip TDDFT (SF-TDDFT). We discuss the pros and cons of each of these approaches in this context. The main conclusion is that these methods complement each other, and provide a reliable alternative to CASPT2, currently considered to be the state-of-the-art reference method for computational photochemistry. ${ }^{[12]}$

\section{Results and Discussion}

Naphthalene radical cation. Naphthalene (Figure 1) is the smallest member of the PAH family. Its presence in monocationic form in the ISM has long been debated. ${ }^{[2,13]} \mathrm{Nap}^{++}$ is known to rapidly regenerate its original ground state form following initial excitation to its lowest bright $D_{2}$ excited state. ${ }^{[4]} A$ CASSCF theoretical study ${ }^{[7]}$ of the $D_{0}, D_{1}$ and $D_{2}$ PESs revealed a sequence of energetically accessible sloped conical intersections combined with electronic state energy gradients pointing directly along their tuning modes. These static features 
account for the ultrafast access to these funnels, which has since been confirmed by quantum dynamical studies. ${ }^{[14]}$

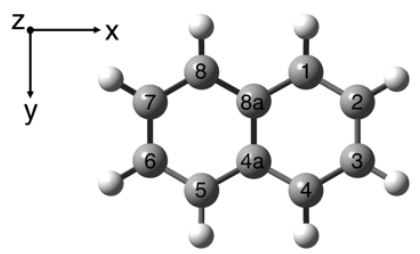

Figure 1. Naphthalene and atom numbering used.

Figure 2 displays the potential energy profiles for the photophysical radiationless decay pathway in $\mathrm{Nap}^{.+}$obtained at different computational levels of theory, including relevant relative energies between critical points. Table 1 provides information about their structures.

All levels of calculation agree on the topological features of the three lowest-energy PESs: one minimum structure of $D_{2 h}$ symmetry is found on each of the $D_{0}, D_{1}$ and $D_{2} P E S s$, and sloped $D_{0} / D_{1}$ and $D_{1} / D_{2}$ minimum energy conical intersections (MECls) are encountered along symmetry-preserving tuning modes which coincide with the main relaxation coordinate of the system in its respective electronic states. The mechanistic picture for the photophysics of $\mathrm{Nap}^{\circ+}$ is therefore consistent across all of these levels of theory, pointing to successive ultrafast nonradiative decay channels from $D_{2}$ to $D_{1}$ and then from $D_{1}$ to $D_{0}$.

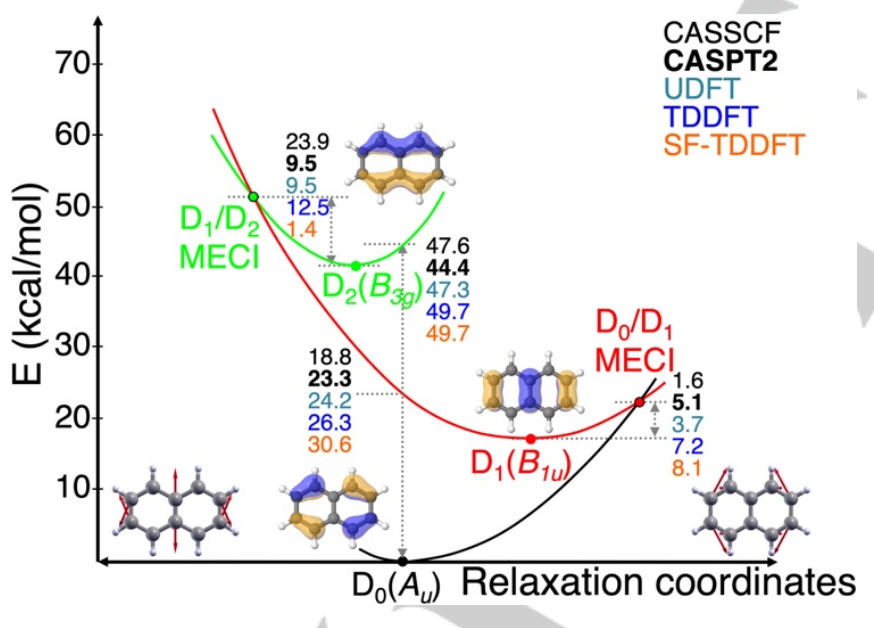

Figure 2. Schematic potential energy profile for the three lowest electronic states of the naphthalene radical cation. The corresponding singly-occupied molecular orbitals are shown for each doublet state. Vertical transition energies to the $D_{1}$ and $D_{2}$ states and activation energies to the crossings are given in $\mathrm{kcal} / \mathrm{mol}$ at various levels of theory.

If we compare in detail the results from the different levels of theory illustrated in Figure 2, there are some notable differences though. While the structures of the $D_{0}, D_{1}$ and $D_{2}$ minima are all in very good agreement (Table 1), larger deviations are observed for the $\mathrm{MECl}$ structures. This is not so surprising, as these geometries are sensitive to the differential correlation energy between the excited states. Figure 2 shows that CASSCF gives a too low $\mathrm{D}_{0}-\mathrm{D}_{1}$ vertical transition energy (by ca. $5 \mathrm{kcal} / \mathrm{mol}$ ), while the value obtained with SF-TDDFT is too high (by ca. $7 \mathrm{kcal} / \mathrm{mol}$ ), compared to the reference CASPT2 value. On the contrary, the $D_{1}-D_{2}$ gap is too high at CASSCF level (by ca. $8 \mathrm{kcal} / \mathrm{mol}$ ) and a bit too low with SF-TDDFT (by ca. 2 $\mathrm{kcal} / \mathrm{mol})$. UDFT is the DFT-based method that gives the closest vertical transition energies to the CASPT2 reference results for this cation. As a consequence, the CASSCF and SF-TDDFT $\mathrm{MECl}$ structures are the ones that deviate furthest from the CASPT2 $\mathrm{MECl}$ geometries, whereas UDFT provides highly accurate geometries (Table 1).

Another consequence of these discrepancies in the differential correlation energies can be seen in terms of energy position of the MECls. As pointed out in ref. [7], the CASSCF $D_{1} / D_{2} \mathrm{MECl}$ lies ca. $24 \mathrm{kcal} / \mathrm{mol}$ above the $\mathrm{D}_{2}$ minimum, which represents a substantial activation energy to reach this funnel, inconsistent with the observed ultrafast ground-state recovery upon photoexcitation to $D_{2}$. The high-energy location of this funnel is a direct outcome of the too-high $\mathrm{D}_{1}-\mathrm{D}_{2}$ energy gap at the CASSCF level. On the contrary, with SF-TDDFT, this crossing is too low in energy relative to the $D_{2}$ minimum. A similar reasoning can be used for the $D_{0} / D_{1} \mathrm{MECl}$, with CASSCF placing the crossing too low in energy with respect to the $D_{1}$ minimum, whereas it is located too high at the SF-TDDFT level. As for the $\mathrm{MECl}$ structures, UDFT pinpoints the two funnels in very good agreement with the CASPT2 results. Nevertheless, overall the DFT-based results consistently describe the ultrafast nonradiative decay pathways responsible for the photophysics of $\mathrm{Nap}^{*+}$.

Table 1. Bond lengths $(\AA)$ in naphthalene radical cation for $D_{0}, D_{1}$ and $D_{2}$ minima and $D_{0} / D_{1}$ and $D_{1} / D_{2}$ MECls at various levels of theory.

\begin{tabular}{|c|c|c|c|c|c|}
\hline Structure & Method & $1-2$ & $2-3$ & $1-8 a$ & $4 a-8 a$ \\
\hline \multirow{5}{*}{$\begin{array}{l}\mathrm{D}_{0}\left({ }^{2} A_{u}\right) \\
\text { minimum }\end{array}$} & CASSCF & 1.407 & 1.388 & 1.409 & 1.432 \\
\hline & CASPT2 & 1.404 & 1.392 & 1.410 & 1.425 \\
\hline & UDFT & 1.406 & 1.392 & 1.414 & 1.433 \\
\hline & $\operatorname{TDDFT}^{[\mathrm{a}]}$ & n.r. & n.r. & n.r. & n.r. \\
\hline & SF-TDDFT & 1.407 & 1.391 & 1.414 & 1.433 \\
\hline \multirow{5}{*}{$\begin{array}{l}\mathrm{D}_{1}\left({ }^{2} \mathrm{~B}_{1 u}\right) \\
\text { minimum }\end{array}$} & CASSCF & 1.368 & 1.452 & 1.421 & 1.467 \\
\hline & CASPT2 & 1.376 & 1.452 & 1.419 & 1.480 \\
\hline & UDFT & 1.372 & 1.454 & 1.419 & 1.483 \\
\hline & TDDFT & 1.371 & 1.456 & 1.418 & 1.484 \\
\hline & SF-TDDFT & 1.370 & 1.456 & 1.419 & 1.481 \\
\hline \multirow{5}{*}{$\begin{array}{l}\mathrm{D}_{2}\left({ }^{2} \mathrm{~B}_{3 g}\right) \\
\text { minimum }\end{array}$} & CASSCF & 1.391 & 1.404 & 1.446 & 1.401 \\
\hline & CASPT2 & 1.392 & 1.408 & 1.451 & 1.405 \\
\hline & UDFT & 1.386 & 1.408 & 1.456 & 1.395 \\
\hline & TDDFT & 1.391 & 1.405 & 1.450 & 1.407 \\
\hline & SF-TDDFT & 1.386 & 1.410 & 1.455 & 1.395 \\
\hline $\mathrm{D}_{0} / \mathrm{D}_{1}$ & CASSCF & 1.361 & 1.465 & 1.423 & 1.475 \\
\hline \multirow[t]{4}{*}{$\mathrm{MECl}$} & CASPT2 & 1.351 & 1.503 & 1.421 & 1.533 \\
\hline & UDFT & 1.349 & 1.504 & 1.421 & 1.529 \\
\hline & TDDFT $^{[b]}$ & 1.358 & 1.485 & 1.421 & 1.510 \\
\hline & SF-TDDFT & 1.337 & 1.533 & 1.421 & 1.547 \\
\hline $\mathrm{D}_{1} / \mathrm{D}_{2}$ & CASSCF & 1.437 & 1.327 & 1.488 & 1.300 \\
\hline \multirow[t]{2}{*}{$\mathrm{MECl}$} & CASPT2 & 1.404 & 1.359 & 1.489 & 1.322 \\
\hline & UDFT & 1.402 & 1.363 & 1.497 & 1.318 \\
\hline
\end{tabular}




\begin{tabular}{lllll} 
TDDFT & 1.418 & 1.349 & 1.494 & 1.326 \\
SF-TDDFT & 1.399 & 1.373 & 1.487 & 1.339 \\
\hline
\end{tabular}

[a] n.r.: not relevant. [b] within the Tamm-Dancoff approximation.

Pyrene radical cation. Pyrene (Figure 3 ) is constituted of four fused benzene rings, and its monocation has often been considered as a potential candidate for DIB carriers. ${ }^{[3]}$ While no time-resolved photophysical study has yet been reported for $\mathrm{Pyr}^{\circ}$, its photodynamics have been investigated with quantum dynamics ${ }^{[5]}$ showing very efficient nonradiative decay from $D_{2}$ down to $D_{0}$ on the picosecond time scale, as observed in $\mathrm{Nap}^{*+}$. In addition, our earlier static investigation ${ }^{[8]}$ of the $D_{0}, D_{1}$ and $D_{2}$ PESs predicted a similar ultrafast ground-state recovery mechanism to the one observed in $\mathrm{Nap}^{\cdot+}$ involving successive sloped $D_{1} / D_{2}$ and $D_{0} / D_{1} M E C l s$.

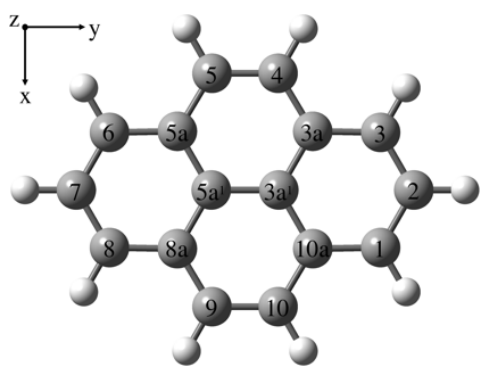

Figure 3. Pyrene and atom numbering used.

The potential energy profiles for the photophysical pathway in $\mathrm{Pyr}^{\circ+}$ are represented in Figure 4. They are qualitatively very similar to the ones shown in Figure 2 for $\mathrm{Nap}^{-+}$, with one minimum structure of $D_{2 h}$ symmetry found on each of the $D_{0}, D_{1}$ and $D_{2}$ PESs, and sloped $D_{0} / D_{1}$ and $D_{1} / D_{2}$ MECls encountered along the main symmetry-preserving relaxation coordinates. All levels of theory used in this work agree with this static picture. (Note that it was not possible to optimize structures at the CASPT2 level for this system, as the large active space required makes the CASPT2 optimizations excessively time-consuming).

The structures reported in Table 2 show that all of the minima for $\mathrm{Pyr}^{\circ+}$ are consistently described at all levels of theory. As was found for $\mathrm{Nap}^{*+}$, larger deviations may be observed at MECls. No reference CASPT2 data are available for this system as yet, but it appears that UDFT and TDDFT provide structures lying between the CASSCF and SF-TDDFT ones. This can be interpreted on the same grounds as for $\mathrm{Nap}^{-+}$. As the CASSCF $\mathrm{D}_{0}-\mathrm{D}_{1}$ energy gap is too small (by ca. $2 \mathrm{kcal} / \mathrm{mol}$ compared to CASPT2), the $D_{0} / D_{1} M E C l$ lies too close to the $D_{1}$ minimum. This is the opposite at the SF-TDDFT level, for which the $D_{0}-D_{1}$ energy gap is too large (by ca. $7 \mathrm{kcal} / \mathrm{mol}$ ) and the $D_{0} / D_{1} \mathrm{MECl}$ is energetically too high with respect to the $D_{1}$ minimum. On the contrary, the CASSCF $D_{1}-D_{2}$ energy gap is too large (by ca. 4 $\mathrm{kcal} / \mathrm{mol}$ ) and, as a result, the $D_{1} / \mathrm{D}_{2} \mathrm{MECl}$ is energetically too high with respect to the $D_{2}$ minimum. With SF-TDDFT, this is the opposite, with a slightly too small $D_{1}-D_{2}$ energy gap (by ca. 1 $\mathrm{kcal} / \mathrm{mol}$ ) resulting in a $D_{1} / \mathrm{D}_{2} \mathrm{MECl}$ lying a bit too close to the $D_{2}$ minimum. As in $\mathrm{Nap}^{*+}$, the best estimate for the structural and energetic positions of the critical points seems to be provided by UDFT, in the absence of CASPT2 reference data.

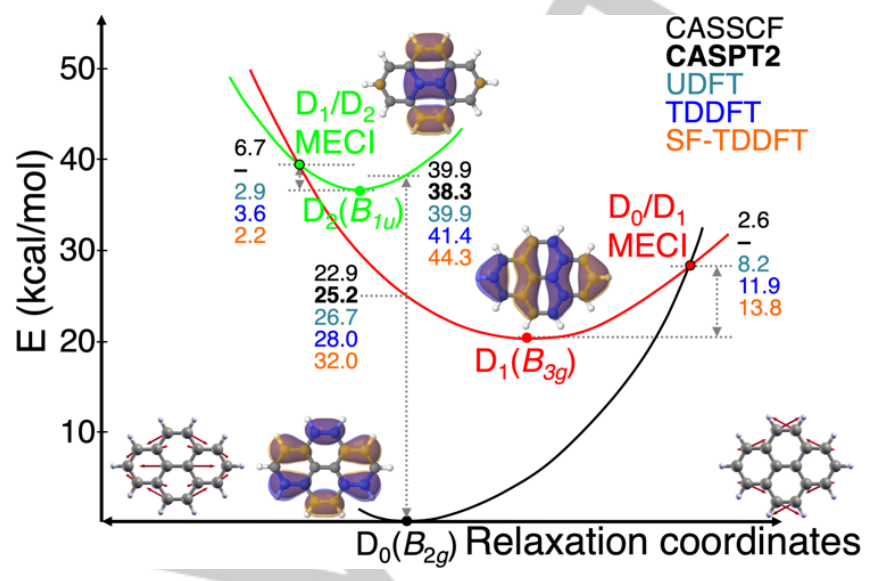

Figure 4. Schematic potential energy profile for the three lowest electronic states of the pyrene radical cation. The corresponding singly-occupied molecular orbitals are shown for each doublet state. Vertical transition energies to the $D_{1}$ and $D_{2}$ states and activation energies to the crossings are given in $\mathrm{kcal} / \mathrm{mol}$ at various levels of theory.

Table 2. Bond lengths $(\AA)$ in pyrene radical cation for $D_{0}, D_{1}$ and $D_{2}$ minima and $D_{0} / D_{1}$ and $D_{1} / D_{2}$ MECls at various levels of theory.

\begin{tabular}{|c|c|c|c|c|c|c|c|}
\hline Structure & Method & $2-3$ & $3-3 a$ & $3 a-4$ & $\begin{array}{l}3 a- \\
3 a^{1}\end{array}$ & $4-5$ & $\begin{array}{l}3 a^{1}- \\
5 a^{1}\end{array}$ \\
\hline $\mathrm{D}_{0}\left({ }^{2} B_{2 g}\right)$ & CASSCF & 1.392 & 1.422 & 1.415 & 1.419 & 1.383 & 1.416 \\
\hline \multirow[t]{4}{*}{ minimum } & CASPT2 & - & - & - & - & - & - \\
\hline & UDFT & 1.393 & 1.423 & 1.418 & 1.425 & 1.385 & 1.417 \\
\hline & TDDFT $^{[a]}$ & n.r. & n.r. & n.r. & n.r. & n.r. & n.r. \\
\hline & SF-TDDFT & 1.393 & 1.422 & 1.418 & 1.425 & 1.385 & 1.417 \\
\hline $\mathrm{D}_{1}\left({ }^{2} \mathrm{~B}_{3 g}\right)$ & CASSCF & 1.406 & 1.385 & 1.450 & 1.446 & 1.353 & 1.395 \\
\hline \multirow[t]{4}{*}{ minimum } & CASPT2 & - & - & - & - & - & - \\
\hline & UDFT & 1.409 & 1.388 & 1.446 & 1.451 & 1.355 & 1.397 \\
\hline & TDDFT & 1.407 & 1.388 & 1.447 & 1.450 & 1.355 & 1.399 \\
\hline & SF-TDDFT & 1.408 & 1.387 & 1.448 & 1.450 & 1.354 & 1.398 \\
\hline $\mathrm{D}_{2}\left({ }^{2} \mathrm{~B}_{1 u}\right)$ & CASSCF & 1.394 & 1.405 & 1.423 & 1.427 & 1.391 & 1.474 \\
\hline \multirow[t]{4}{*}{ minimum } & CASPT2 & - & - & - & - & - & - \\
\hline & UDFT & 1.394 & 1.404 & 1.430 & 1.428 & 1.389 & 1.482 \\
\hline & TDDFT & 1.394 & 1.405 & 1.428 & 1.430 & 1.389 & 1.478 \\
\hline & SF-TDDFT & 1.394 & 1.403 & 1.432 & 1.428 & 1.388 & 1.485 \\
\hline $\mathrm{D}_{0} / \mathrm{D}_{1}$ & CASSCF & 1.413 & 1.369 & 1.467 & 1.458 & 1.339 & 1.385 \\
\hline \multirow[t]{4}{*}{$\mathrm{MECl}$} & CASPT2 & - & - & - & - & - & - \\
\hline & UDFT & 1.426 & 1.353 & 1.481 & 1.482 & 1.322 & 1.375 \\
\hline & TDDFT $^{[b]}$ & 1.424 & 1.361 & 1.472 & 1.477 & 1.329 & 1.378 \\
\hline & SF-TDDFT & 1.430 & 1.343 & 1.496 & 1.490 & 1.310 & 1.369 \\
\hline $\mathrm{D}_{1} / \mathrm{D}_{2}$ & CASSCF & 1.382 & 1.424 & 1.400 & 1.413 & 1.428 & 1.543 \\
\hline \multirow[t]{4}{*}{$\mathrm{MECl}$} & CASPT2 & - & - & - & - & - & - \\
\hline & UDFT & 1.384 & 1.414 & 1.420 & 1.415 & 1.413 & 1.541 \\
\hline & TDDFT & 1.383 & 1.418 & 1.414 & 1.416 & 1.417 & 1.542 \\
\hline & SF-TDDFT & 1.385 & 1.411 & 1.424 & 1.416 & 1.411 & 1.536 \\
\hline
\end{tabular}

[a] n.r.: not relevant. [b] within the Tamm-Dancoff approximation. 
Table 3. Bond lengths $(\AA)$ in perylene radical cation for $D_{2 h} D_{0}, D_{1}$ and $D_{2}$ minima and $D_{0} / D_{1}$ and $D_{1} / D_{2}$ MECls at various levels of theory.

\begin{tabular}{|c|c|c|c|c|c|c|c|c|}
\hline Structure & Method & $1-2$ & $2-3$ & $3-3 a$ & $3 a-3 a^{1}$ & $3 a^{1}-6 a$ & $6-6 a$ & $6 a-6 b$ \\
\hline \multirow{4}{*}{$\begin{array}{l}\mathrm{D}_{0}\left({ }^{2} A_{u}\right) \\
\text { minimum }\end{array}$} & RASSCF[a] & 1.389 & 1.382 & 1.411 & 1.428 & 1.421 & 1.410 & 1.446 \\
\hline & UDFT & 1.393 & 1.388 & 1.414 & 1.433 & 1.429 & 1.412 & 1.452 \\
\hline & $\mathrm{TDDFT}^{[\mathrm{b}]}$ & n.r. & n.r. & n.r. & n.r. & n.r. & n.r. & n.r. \\
\hline & SF-TDDFT & 1.393 & 1.388 & 1.414 & 1.432 & 1.429 & 1.412 & 1.452 \\
\hline \multirow{4}{*}{$\begin{array}{l}\mathrm{D}_{1}\left({ }^{2} \mathrm{~B}_{3 g}\right) \\
\text { minimum }\end{array}$} & RASSCF $[\mathrm{a}]$ & 1.368 & 1.406 & 1.407 & 1.430 & 1.420 & 1.436 & 1.433 \\
\hline & UDFT & 1.372 & 1.409 & 1.410 & 1.436 & 1.426 & 1.453 & 1.417 \\
\hline & TDDFT & 1.379 & 1.406 & 1.412 & 1.435 & 1.427 & 1.434 & 1.440 \\
\hline & SF-TDDFT & 1.372 & 1.409 & 1.410 & 1.436 & 1.426 & 1.453 & 1.416 \\
\hline \multirow{4}{*}{$\begin{array}{l}\mathrm{D}_{1}\left({ }^{2} \mathrm{~B}_{2 g}\right) \\
\text { minimum }\end{array}$} & RASSCF[a] & 1.426 & 1.360 & 1.421 & 1.444 & 1.423 & 1.387 & 1.468 \\
\hline & UDFT & 1.424 & 1.374 & 1.415 & 1.461 & 1.432 & 1.386 & 1.475 \\
\hline & TDDFT & 1.425 & 1.372 & 1.416 & 1.460 & 1.431 & 1.387 & 1.474 \\
\hline & SF-TDDFT & 1.424 & 1.379 & 1.405 & 1.475 & 1.434 & 1.378 & 1.483 \\
\hline $\mathrm{D}_{0} / \mathrm{D}_{1}\left({ }^{2} \mathrm{~B}_{2 g}\right)$ & MMVB $^{[a]}$ & 1.592 & 1.327 & 1.438 & 1.466 & 1.436 & 1.333 & 1.563 \\
\hline \multirow{2}{*}{$\mathrm{MECl}$} & $\mathrm{TDDFT}^{[\mathrm{c}]}$ & 1.553 & 1.327 & 1.419 & 1.600 & 1.433 & 1.308 & 1.563 \\
\hline & SF-TDDFT & 1.573 & 1.322 & 1.417 & 1.618 & 1.431 & 1.293 & 1.592 \\
\hline \multirow[t]{4}{*}{$\mathrm{D}_{1} / \mathrm{D}_{2} \mathrm{MECl}$} & MMVB $^{[a]}$ & 1.419 & 1.386 & 1.425 & 1.439 & 1.431 & 1.423 & 1.455 \\
\hline & UDFT & 1.393 & 1.398 & 1.415 & 1.425 & 1.426 & 1.399 & 1.518 \\
\hline & TDDFT & 1.399 & 1.392 & 1.413 & 1.445 & 1.429 & 1.410 & 1.461 \\
\hline & SF-TDDFT & 1.394 & 1.394 & 1.410 & 1.448 & 1.436 & 1.415 & 1.443 \\
\hline
\end{tabular}

[a] Taken from Ref. [9]. [b] n.r.: not relevant. [c] within the Tamm-Dancoff approximation.

Perylene radical cation. The perylene (Figure 5) cation, which is made up of five fused benzene rings, presents a very different photophysical behavior compared to $\mathrm{Nap}^{\cdot+}$ and $\mathrm{Pyr}^{++}$. While these two cations present an ultrafast ground-state recovery on a sub-ps timescale, the excited-state lifetime in Per ${ }^{-+}$is at least an order of magnitude longer (ps timescale). ${ }^{[4]} \mathrm{Per}^{++}$is also the smallest $\mathrm{PAH}$ cation to date displaying fluorescence from its lowest $D_{1}$ excited state. ${ }^{[6]}$ This has been accounted for by the absence of accessible funnels between $D_{1}$ and $D_{0} \cdot{ }^{\left[{ }^{[9]}\right.}$ Because of the size of the system, these funnels were sought using the Molecular Mechanics - Valence Bond (MMVB) hybrid method, which incorporates a parameterized effective Hamiltonian, allowing it to reproduce CASSCF results reasonably accurately at a fractional cost. ${ }^{[15]}$ Here we show that the potential energy landscape of $\mathrm{Per}^{*+}$ - including relevant conical intersections - can be investigated with DFT-based approaches.

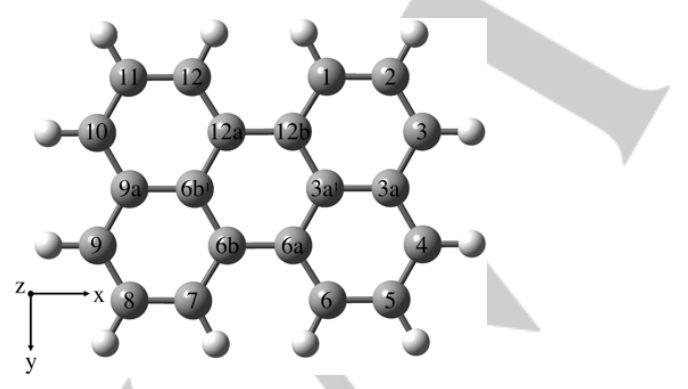

Figure 5. Perylene and atom numbering used.

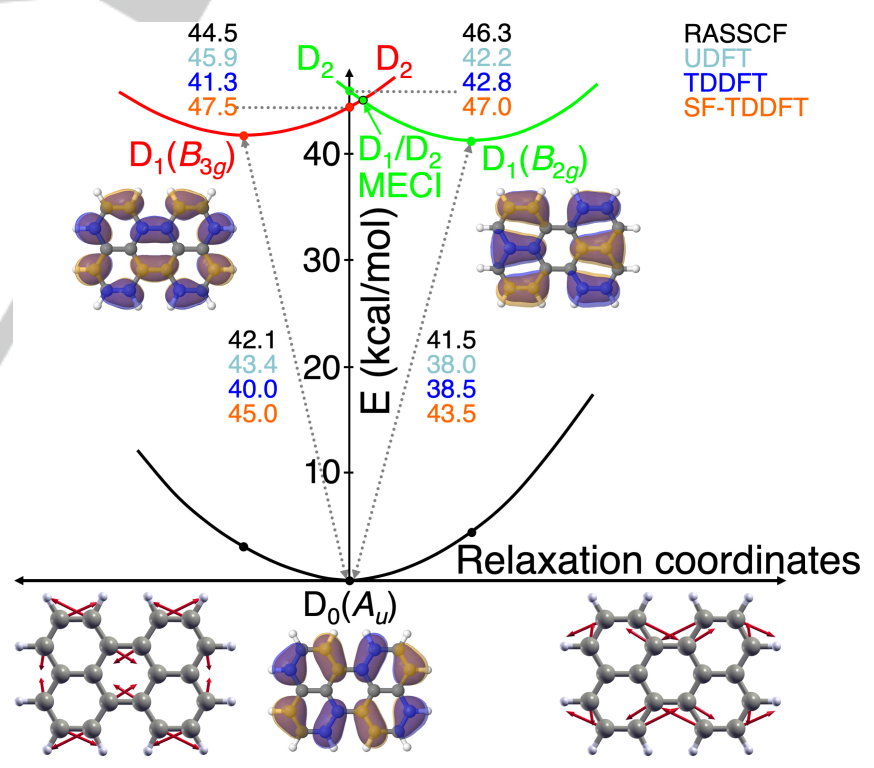

Figure 6. Schematic potential energy profile for the three lowest electronic states of the perylene radical cation. The corresponding singly-occupied molecular orbitals are shown for each doublet state. Vertical and adiabatic transition energies to the $D_{1}$ and $D_{2}$ states are given in $\mathrm{kcal} / \mathrm{mol}$ at various levels of theory.

Figure 6 shows the potential energy landscapes for the lowest three electronic states of $\mathrm{Per}^{++}$. One of the main differences compared with $\mathrm{Nap}^{\cdot+}$ and $\mathrm{Pyr}^{*+}$ is the large $\mathrm{D}_{0}-\mathrm{D}_{1}$ energy gap: while it is ca. $25 \mathrm{kcal} / \mathrm{mol}$ in $\mathrm{Nap}^{++}$and $\mathrm{Pyr}^{++}$, it is over 40 
$\mathrm{kcal} / \mathrm{mol}$ in Per ${ }^{*+}$. Another difference is that $D_{2}$ lies very close to $D_{1}$ at the $D_{0}$ equilibrium geometry. Consequently, while RASSCF and TDDFT predict $D_{1}$ and $D_{2}$ to be the $B_{3 g}$ and $B_{2 g}$ states respectively, UDFT and SF-TDDFT give the reverse order. There is thus some ambiguity regarding the assignment of the lowest two excited states in Per ${ }^{++}$but what is known is that the two states are very close in energy, and cross at an $\mathrm{MECl}$ in the Franck-Condon region, effectively forming a single adiabatic $D_{1}$ PES together. Due to the peaked topology of the $D_{1} / D_{2}$ conical intersection in $\mathrm{Per}^{++}$(also contrasting with $\mathrm{Nap}^{\cdot+}$ and $\mathrm{Pyr}^{\cdot+}$ ), each state has its own minimum on the $D_{1}$ adiabatic PES following symmetry-preserving in-plane relaxation, as shown in Figure 6. In fact, the $D_{1}\left(B_{2 g}\right)$ minimum on the PES was found to belong to the $C_{2 v}$ symmetry point group, but with zero-point energy taken into account, it becomes $D_{2 h}$ symmetry. ${ }^{[9]}$ Thus, we only report the $D_{2 h}$ optimized structure in Table 3 in this study; the minimum in this higher symmetry.

All levels of theory used here agree with these topological features of the $D_{1}$ and $D_{2}$ PESs. The structures of the minima are all in good agreement (Table 3). It is worth noting though that the $B_{3 g}$ state presents a multiconfigurational character, mixing a dominant $\pi \rightarrow \pi^{*}$ configuration with a $\pi \rightarrow \pi$ configuration. ${ }^{[9]}$ This multiconfigurational description of the excited-state wavefunction is well captured at the TDDFT level, while SF-TDDFT and UDFT describe only the main $\pi \rightarrow \pi^{*}$ configuration. This possibly explains the slightly better agreement between the RASSCF and TDDFT structures for the corresponding $D_{1}\left(B_{3 g}\right)$ minimum with respect to the SF-TDDFT and UDFT ones, for which larger deviations are observed for the $6-6 a$ and $6 a-6 b$ bond lengths. Regarding the $D_{1}\left(B_{2 g}\right)$ minimum, its optimization proved to be difficult at the SF-TDDFT level. We could only optimize the structure close to a stationary point, possibly due to an instability in the reference quartet state wavefunction.

The observed luminescence of $\mathrm{Per}^{\circ+}$ was previously explained by the absence of accessible $D_{0} / D_{1}$ crossings, based on an extensive search for them with the computationally efficient MMVB approach..$^{[9,15]}$ Three different $D_{0} / D_{1}$ MECls were located at higher energies with MMVB: one involving a crossing with the $D_{1}\left(B_{2 g}\right)$ state with a $D_{2 h}$ structure lying at $41 \mathrm{kcal} / \mathrm{mol}$ above the $\mathrm{D}_{1}\left(B_{2 g}\right)$ minimum. This structure shows unusually stretched bonds 1-2 and 6a-6b and compressed bonds 2-3 and 6-6a (see Table 3). A lower-energy $\mathrm{MECl}$ with $C_{2 v}$ symmetry involving the same excited state was also found, still lying $28 \mathrm{kcal} / \mathrm{mol}$ above the $D_{1}\left(B_{2 g}\right)$ minimum. Finally, a third $\mathrm{MECl}$ involving the $\mathrm{D}_{1}\left(B_{3 g}\right)$ excited state was located at over $80 \mathrm{kcal} / \mathrm{mol}$ above the $D_{1}$ minimum. These funnels cannot provide efficient radiationless decay routes, due to the high activation energies required to reach them. We tested this conclusion using our UDFT, TDDFT and SF-TDDFT computational protocol to locate the first two crossings (the third one - being very high in energy - was not searched for). The structures for the $D_{0} / D_{1}\left(B_{2 g}\right) D_{2 h} \mathrm{MECl}$ at different levels of theory are given in Table 3 . They all agree with MMVB in displaying long 1-2 and 6a-6b bonds and short 2-3 and 6-6a bonds. However, the DFT-based calculations also predict a very long $3 a-3 a^{1}$ bond, at variance with MMVB. This $\mathrm{MECl}$ is located at $64.8,55.3$ and $74.4 \mathrm{kcal} / \mathrm{mol}$ above the $\mathrm{D}_{1}$ minimum with UDFT, TDDFT and SF-TDDFT, respectively. The lower $C_{2 v}$ $\mathrm{D}_{0} / \mathrm{D}_{1} \mathrm{MECl}$ structures are shown in Figure 7 and are characterized by very long $1-2$ and $3 a-3 a^{1}$ bonds (1.58 to 1.77 $\AA$ ) and very short $2-3,6-6 a, 6 b-7$ and $8-9$ bonds (1.36 to $1.30 \AA$ ). The main disagreement with MMVB concerns the 6-6a bond length, which is predicted to be ca. $0.1 \AA$ longer than all of the DFT-based calculations. The $D_{0} / D_{1} C_{2 v} \mathrm{MECl}$ is located at 56.4 , 43.2 and $61.1 \mathrm{kcal} / \mathrm{mol}$ above the $D_{1}$ minimum at the UDFT, TDDFT and SF-TDDFT levels, respectively. This is substantially higher than the activation energy predicted at the MMVB level. All of these DFT-based results nevertheless confirm the inaccessibility of $D_{0} / D_{1}$ funnels in Per ${ }^{*+}$, leaving radiative decay / fluorescence as the most probable photophysical pathway in this case.

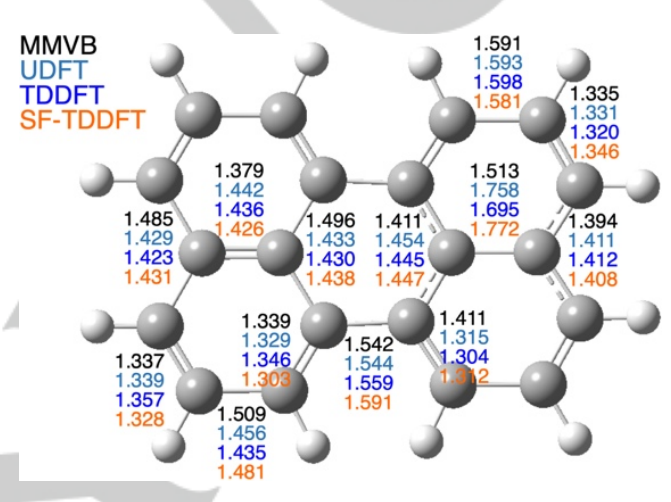

Figure 7. The inaccessible $D_{0} / D_{1} \quad C_{2 v} \mathrm{MECl}$ structure in perylene cation optimized at various levels of theory.

\section{Conclusions}

We have shown that DFT-based approaches such as UDFT, TDDFT and SF-TDDFT allow us to investigate the potential energy landscapes of PAH cations, including the location of the critical conical intersections that are intimately connected to the photophysical behavior of these systems.

The ultrafast ground-state recoveries in $\mathrm{Nap}^{++}$and $\mathrm{Pyr}^{++}$are accounted for with UDFT, TDDFT and SF-TDDFT, which all locate easily accessible sloped $D_{1} / D_{2}$ and $D_{0} / D_{1}$ conical intersections. On the other hand, the longer excited-state lifetime in $\mathrm{Per}^{\circ+}$ (as well as the observed fluorescence) are explained by the absence of any low-lying $D_{0} / D_{1}$ conical intersections. Energies and geometries obtained with the DFT-based methods used here compare well with the available ab initio reference calculations, including those for conical intersections.

There are some cautions though. UDFT can only be used if the ground and excited states belong to different electronic symmetries, ${ }^{[16]}$ although there is no such restriction with TDDFT and SF-TDDFT, which can be applied to systems with no particular symmetry. Note that, in such cases, TDDFT does not couple the ground and lowest excited state properly. While this is not an issue in SF-TDDFT, this method depends on having a suitable unrestricted reference state (here a quartet state) to generate (by spin-flip) single-electron transitions the desired doublet states of the radical cations. 
With these provisos, we believe that the DFT-based strategies tested and validated in this work to describe the photophysical behavior of the naphthalene, pyrene and perylene radical cations offer a promising tool to investigate the photophysics of much larger $\mathrm{PAH}$ cations and of other relevant radicals, ${ }^{[17]}$ for which ab initio multiconfigurational wavefunction-based approaches become prohibitively expensive.

\section{Computational Section}

UDFT, ${ }^{[18]}$ TDDFT $^{[19]}$ and SF-TDDFT ${ }^{[20]}$ geometry optimizations of critical structures (minima and conical intersections) were all performed using the B3LYP functional[ ${ }^{[21]}$ together with the $6-31 \mathrm{G}^{*}$ orbital basis ${ }^{[22]}$ set. SFTDDFT calculations of doublet states require using a suitable reference quartet state. ${ }^{[23]}$ All CASSCF calculations were carried out using the full valence $\pi$ active space, i.e. $(9 e, 100)$ for $\mathrm{Nap}^{*+}$ and $(15 \mathrm{e}, 160)$ for $\mathrm{Pyr}^{++}$ along with the same $6-31 \mathrm{G}^{*}$ basis set. CASPT2 calculations for $\mathrm{Nap}^{++}$ and $\mathrm{Pyr}^{+}$were performed using the reference $\operatorname{CASSCF}(9,10)$ and $\operatorname{CASSCF}(15,16)$ wavefunctions with a correlation-consistent cc-pVTZ basis ${ }^{[24]}$ set. The Cholesky decomposition ${ }^{[25]}$ with a threshold of $10^{-6}$ a.u. and an imaginary shift ${ }^{[26]}$ of 0.1 a.u. was used in all of the CASPT2 calculations. Analytical energy gradients were computed for UDFT, TDDFT, SF-TDDFT and CASSCF, while numerical energy gradients were evaluated with CASPT2. CASPT2 geometry optimizations remain prohibitively expensive in $\mathrm{Pyr}^{++}$for which we only calculated the vertical transition energies from the $D_{0}$ geometry optimized at the CASSCF level. Different algorithms were used to locate MECls: either based on a gradient projection method (for CASSCF); on a Lagrange multiplier method (UDFT and CASPT2); or on a penalty function method (TDDFT and SF-TDDFT). Note that the Tamm-Dancoff approximation (TDA) was used to locate the $D_{0} / D_{1}$ crossings to avoid imaginary roots in the random-phase approximation (RPA) near conical intersections. Calculations were performed using different quantum chemistry codes, depending on methods currently implemented therein: Gaussian 09, ${ }^{[27]}$ Molpro, ${ }^{[28]}$ Molcas $^{[29]}$ and QChem. ${ }^{[30]}$

\section{Acknowledgements}

This work was granted access to the HPC resources of CALMIP supercomputing center under the allocation 2019-[12158]. MBP thanks David Sanchez for the installation of QChem. The authors thank Christine Joblin and Fernand Spiegelman for their support and helpful discussions.

Keywords: PAH • DFT • TD-DFT • Photophysics • conical intersection

[1] a) G. P. van der Zwet, L. J. Allamandola, Astron. Astrophys. 1985, 146, 76-80; b) A. Léger, L. d'Hendecourt, Astron. Astrophys. 1985, 146, $81-$ 85 ; c) L. J. Allamandola, A. G. G. M. Tielens, J. R. Barker, Astrophys. J. 1985, 290, L25-L28.

[2] a) F. Salama, L. J. Allamandola, Astrophys. J. 1992, 395, 301-306; b) T. P. Snow, Astrophys. J. 1992, 401, 775-777; c) S. Iglesias-Groth, A. Manchado, D. A. García-Hernández, J. I. González Hernández, D. L. Lambert, Astrophys. J. 2008, 685, L55-L58; d) S. Iglesias-Groth, J. González Hernández, A. Manchado, Mon. Not. R. Astron. Soc. 2012, 420, 2785-2792.

[3] a) O. Parisel, G. Berthier, Y. Ellinger, Astron. Astrophys. 1992, 266, L1L4; b) F. Salama, L. J. Allamandola, Nature 1992, 358, 42-43; c) F.
Salama, L. J. Allamandola, J. Chem. Soc. Faraday Trans. 1993, 89, 2277-2284; d) J. Szczepanski, M. Vala, Nature 1993, 363, 699-701.

[4] L. Zhao, R. Lian, I. A. Shkrob, R. A. Crowell, S. Pommeret, E. L. Chronister, A. D. Liu, A. D. Trifunac, J. Phys. Chem. A 2004, 108, 2531.

[5] S. N. Reddy, S. Mahapatra, J. Phys. Chem. B 2015, 119, 11391-11402.

[6] a) C. Joblin, F. Salama, L. Allamandola, J. Chem. Phys. 1995, 102, 9743-9745; b) X. D. F. Chillier, B. M. Stone, C. Joblin, F. Salama, L. J. Allamandola, J. Chem. Phys. 2002, 116, 5725-5730.

[7] K. F. Hall, M. Boggio-Pasqua, M. J. Bearpark, M. A. Robb, J. Phys. Chem. A 2006, 110, 13591-13599.

[8] A. M. Tokmachev, M. Boggio-Pasqua, M. J. Bearpark, M. A. Robb, J. Phys. Chem. A 2008, 112, 10881-10886.

[9] A. M. Tokmachev, M. Boggio-Pasqua, D. Mendive-Tapia, M. J. Bearpark, M. A. Robb, J. Chem. Phys. 2010, 132, 044306.

[10] a) F. Krausbeck, D. Mendive-Tapia, A. J. W. Thom, M. J. Bearpark, Comput. Theor. Chem. 2014, 1040-1041, 14-19; b) M. J. Bearpark, F. Ogliaro, T. Vreven, M. Boggio-Pasqua, M. J. Frisch, S. M. Larkin, M. Morrison, M. A. Robb, J. Photochem. Photobiol. A 2007, 190, 207-227.

[11] F.-X. Hardy, C. A. Rice, J. P. Maier, Astrophys. J. 2017, 836, 37.

[12] a) D. Roca-Sanjuán, F. Aquilante, R. Lindh, WIREs Comput. Mol. Sci. 2012, 2, 585-603; b) L. González, D. Escudero, L. Serrano-Andrés, ChemPhysChem 2012, 13, 28-51; c) H. Lischka, D. Nachtigallová, A. J. A. Aquino, P. G. Szalay, F. Plasser, F. B. C. Machado, M. Barbatti, Chem. Rev. 2018, 118, 7293-7361.

[13] a) J. Krelowski, G. A. Galazutdinov, F. A. Musaev, J. Nirski, Mon. Not. R. Astron. Soc. 2001, 328, 810-814; b) G. A. Galazutdinov, B.-C. Lee, I.-O. Song, M. Kazmierczak, J. Krelowski, Mon. Not. R. Astron. Soc. 2011, 412, 1259-1264; c) J. M. Searles, J. D. Destree, T. P. Snow, F. Salama, D. G. York, J. Dahlstrom, J. Astrophys. J. 2011, 732, 50.

[14] a) V. S. Reddy, S. Mahapatra, J. Chem. Phys. 2008, 128, 091104; b) V. S. Reddy, S. Ghanta, S. Mahapatra, Phys. Rev. Lett. 2010, 104, 111102; c) S. Ghanta, V. S. Reddy, S. Mahapatra, Phys. Chem. Chem. Phys. 2011, 13, 14531-14541.

[15] a) F. Bernardi, M. Olivucci, M. A. Robb, J. Am. Chem. Soc. 1992, 114, 1606-1616; b) M. J. Bearpark, F. Bernardi, M. Olivucci, M. A. Robb, J. Phys. Chem. A 1997, 101, 8395-8401; c) M. J. Bearpark, M. BoggioPasqua, Theor. Chem. Acc. 2003, 110 105-114; d) M. J. Bearpark, M. Boggio-Pasqua, M. A. Robb, F. Ogliaro, Theor. Chem. Acc. 2006, 116, 670-682; e) K. F. Hall, A. M. Tokmachev, M. J. Bearpark, M. BoggioPasqua, M. A. Robb, J. Chem. Phys. 2007, 127, 134111

[16] C. A. Jiménez-Hoyos, R. Rodríguez-Guzmán, G. E. Scuseria, J. Chem. Phys. 2013, 139, 224110.

[17] J. Soler, R. Sarkar, M. Boggio-Pasqua, J. Phys. Chem. A 2019, 123, 1824-1829.

[18] R. M. Dreizler, E. K. U. Gross, Density Functional Theory: An Approach to the Quantum Many-Body Problem, Springer-Verlag, New York, 1990, pp. 1-303.

[19] a) M. A. L. Marques, E. K. U. Gross, Annu. Rev. Phys. Chem. 2004, 55, 427-455; b) M. E. Casida, M. Huix-Rotllant, Annu. Rev. Phys. Chem. 2012, 63, 287-323.

[20] Y. Shao, M. Head-Gordon, A. I. Krylov, J. Chem. Phys. 2003, 118, 4807-4818.

[21] a) A. D. Becke, J. Chem. Phys. 1993, 98, 5648-5652; b) P. J. Stephens, F. J. Devlin, C. F. Chabalowski, M. J. Frisch, J. Phys. Chem. 1994, 98, $11623-11627$

[22] a) W. J. Hehre, R. Ditchfield, J. A. Pople, J. Chem. Phys. 1972, 56, 2257-2261; b) P. C. Hariharan, J. A. Pople, Theoret. Chim. Acta 1973 $28,213-222$.

[23] a) Z. Li, W. Liu, J. Chem. Phys. 2012, 136, 024107; b) J. M. Herbert, X Zhang, A. F. Morrison, J. Liu, Acc. Chem. Res. 2016, 49, 931-941.

[24] T. H. Dunning, J. Chem. Phys. 1989, 90, 1007-1023.

[25] a) F. Aquilante, T. B. Pedersen, R. Lindh, J. Chem. Phys. 2007, 126, 194106; b) F. Aquilante, P.-A. Malmqvist, T. B. Pedersen, A. Ghosh, B. O. Roos, J. Chem. Theory Comput. 2008, 4, 694-702; c) J. Boström, M. 
G. Delcey, F. Aquilante, L. Serrano-Andrés, T. B. Pedersen, R. Lindh, J. Chem. Theory Comput. 2010, 6, 747-754.

[26] N. Forsberg, P.-Å. Malmqvist, Chem. Phys. Lett. 1997, 274, 196-204.

[27] M. J. Frisch, G. W. Trucks, H. B. Schlegel, G. E. Scuseria, M. A. Robb, J. R. Cheeseman, G. Scalmani, V. Barone, B. Mennucci, G. A. Petersson, et al., Gaussian 09, revision D.01, Gaussian, Inc., Wallingford CT, 2009.

[28] H.-J. Werner, P. J. Knowles, G. Knizia, F. R. Manby, M. Schütz, WIREs Comput. Mol. Sci. 2012, 2, 242-253.

[29] F. Aquilante, J. Autschbach, R. K. Carlson, L. F. Chibotaru, M. G. Delcey, L. De Vico, I. F. Galván, N. Ferré, L. M. Frutos, L. Gagliardi, M.
Garavelli, A. Giussani, C. E. Hoyer, G. L. Manni, H. Lischka, D. Ma, P. Å. Malmqvist, T. Müller, A. Nenov, M. Olivucci, T. B. Pedersen, D. Peng, F. Plasser, B. Pritchard, M. Reiher, I. Rivalta, I. Schapiro, J. SegarraMartí, M. Stenrup, D. G. Truhlar, L. Ungur, A. Valentini, S. Vancoillie, V. Veryazov, V. P. Vysotskiy, O. Weingart, F. Zapata, R. Lindh, J. Comput. Chem. 2016, 37, 506-541.

[30] Y. S. Shao, Z. Gan, E. Epifanovsky, A. T. B. Gilbert, M. Wormit, J. Kussmann, A. W. Lange, A. Behn, J. Deng, X. Feng, et al., Mol. Phys. 2015, 113, 184-215. 


\section{Entry for the Table of Contents}

\section{ARTICLE}

The photophysics of several smaller polycyclic aromatic hydrocarbon (PAH) cations - naphthalene, pyrene and perylene - is investigated with density functional theory (DFT)-based approaches: unrestricted DFT, timedependent DFT and spin-flip DFT. A consistent mechanistic photophysical picture is obtained compared to accurate multiconfigurational wavefunction-based ab initio methods, in particular with regard to the description of critical conical intersections.

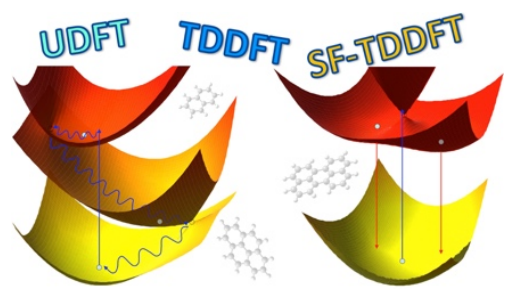

M. Boggio-Pasqua*, M. J. Bearpark

Page No. - Page No.

On the use of density functional theory-based methods to investigate the photophysics of polycyclic aromatic hydrocarbon radical cations: A benchmark study on naphthalene, pyrene and perylene cations. 